\title{
THREE-DIMENSIONAL EVALUATION OF PHARYNGEAL AIRWAY VOL- UME FOLLOWING TREATMENT OF POST-PUBERTAL PATIENTS WITH SKELETAL CLASS II MALOCCLUSION VIA MINIPLATE-ANCHORED HERBST APPLIANCE
}

\author{
Khaled Samy ElHabbak*, Farouk Hussain ${ }^{* *}$, Al-Dany Atwa Al-Dany ${ }^{* * *}$
}

\begin{abstract}
Objective: To evaluate the pharyngeal airway volume following treatment of skeletal Class II mandibular retrusion with miniplate-anchored Herbst appliance by Cone Beam Computed Tomography (СBCT).

Materials and Methods: Fourteen female Class II post-pubertal patients with mandibular retrusion were randomly selected with mean age was $16.7 \pm 1.56$ years. As a first orthodontic phase, they were treated by type IV Herbst functional appliance that anchored by two reconstructive plates located bilaterally in the mandible between the canine and first premolar teeth, while attached to the maxilla through dental splints utilizing orthodontic bands. The CBCTscans were obtained before treatment (T1) and immediately after removal of Herbst appliance (T2) for volumetric pharyngeal airway evaluation. In addition, sagittal cephalometric parameters were analyzed.
\end{abstract}

Results:Paired sample t-testshowed a non-statistically significant $(\mathrm{p}>0.05)$ increase $(585 \mathrm{~mm} 3)$ in nasopharyngeal airway volume with treatment, whereas velopharyngeal airway, glossopharyngeal airway and total airway volume showed statistically significant $(\mathrm{p}<0.01)$ increases after Herbst appliance treatment by $2448 \mathrm{~mm}^{3}, 1107 \mathrm{~mm}^{3}, 2607 \mathrm{~mm}^{3}$, respectively. Moreover, an improvement in mandibular retrognathism was evident as demonstrated by significant increase in SNB angle and significant decrease in ANB angle $(\mathrm{p}<0.05)$.

Conclusions: Pharyngeal airway volume considerably enhanced subsequent to mandibular advancement through miniplateanchored Herbst appliance which could be a helpful treatment opportunity for post-pubertal patients who have skeletal Class II malocclusion with mandibular retusion and suffering breathing disorders.

KEYWORDS: Skeletal Class II; Pharyngeal airway; Herbst functional appliance; Skeletal Anchorage; Miniplates.

\section{INTRODUCTION}

Skeletal Class II malocclusion is one of the most frequent orthodontic problems that constitutes a significant percentage of orthodontic patients ranging from $18 \%$ up to approximately $32 \%$. Despite the variability among Class II patients, were many skeletal and dental combinations donate for the conception of this problem, mandibular retrusion is the foremost contributing factor ${ }^{(1)}$.In management of those growing patients, many clinicians seek to encourage or redirect the mandibular growth to

* Doctorate Candidate, Orthodontic Department, Faculty of Dental Medicine, Al-Azhar University Cairo-boys, Egypt

** Assistant Professor, Department of Orthodontics, Faculty of Dental, Al Azhar University.

*** Department of Orthodontics, Faculty of Dental Medicine, (Boys, Cairo), Al-Azhar University

- Corresponding author: khaledhabbak@gmail.com

DOI: $10.21608 /$ ajdsm.2020.24966.1068 
correct a skeletal Class II malocclusion through the use of functional appliances either removable or fixed to correct this discrepancy ${ }^{(2)}$.

The relationship between pharyngeal, dentofacial, and craniofacial structures have been given much orthodontic concernsince mandibular retrusion has been associated with a decreased oropharyngeal airway dimension, which may be due to posterior positioning of the tongue and hyoid bone $^{(3-5)}$. In addition, it is believed that upper airway constriction is the major source of sleeping disorders, such as snoring and obstructive sleep apnea $(\mathrm{OSA})^{(6)}$. Presently, mandibular anterior repositioning appliances are used for OSA patients to relocate tongue, soft palate and hyoid bone to increase pharyngeal airway dimensions, and subsequently improve respiratory function ${ }^{(7)}$.

The Herbst appliance is a fixed functional bitejumping tool for treating Class II malocclusion to improve the sagittal mandibular position relative to the maxilla and skeletal Class II antero-posterior discrepancy, with a main advantage relatively short period of active treatment and little or no patient cooperation required. ${ }^{(2,8)}$ Because the pharyngeal airway could be expanded anteriorly as a secondary effect of Herbst appliance, improvement of pharyngeal airway obstruction might be expected ${ }^{(9,10)}$.

The original Herbst appliance consisted of banded teeth as an anchorage, unfortunately, undesirable tooth movements and anchorage loss confuse the treatment outcomes of fixed functional appliances (FFAs). ${ }^{(2)}$ Several attempts were proposed to counteract the unwanted dentoalveolar side effects of FFAs, like the use of skeletal anchorage. It was claimed that mini-screw anchored FFAs reduced mandibular incisor proclination but was not able to enhance the skeletal changes. ${ }^{(11-13)}$ Alternatively, miniplates were shown to be well accepted by patients and became to some extent popular for use in various applications. Lately, they were used for direct loading of FFAs for correction of skeletal Class II malocclusion ${ }^{(14-16)}$.
Indeed, the effects of fictional appliances concerning the upper airway have been widely investigated during Class II treatment via conventional radiographic methods, mostly, in pre-pubertal patients $^{(9,17-22)}$. Nevertheless, these radiographic twodimensional (2D) images do not provide information regarding cross-sectional areas or volumes of the upper airway. The introduction of cone-beam computed tomography (CBCT) has enabled detailed three-dimensional (3D) evaluation of the airway, with the benefit of low radiation dose and shorter scanning time compared to $\mathrm{CT}$ and magnetic resonance imaging, respectively ${ }^{(10,23,24)}$.

Yet, little information is available regarding the clinical outcomes concerning the pharyngeal airway dimensions followingskeletally-anchored Herbst appliance at post-pubertal stage. Therefore, the present study was designed to estimate the volumetric pharyngeal airway dimensions after utilizing miniplate-anchored Herbst for treatment of skeletal Class II post-pubertal patients.

\section{MATERIALS AND METHODS}

\section{Study design, setting, sample size estimate, and eligibility criteria:}

The study protocol was reviewed and approved by Institutional Review Board and the Ethical Research Committee of Al-Azhar University, Cairo, Egypt (Approval No. 0000011 on 11/7/2017).The current prospective clinical study was conducted on 14 female patients with an age ranged from 15 to 20 years with mean age was $16.7 \pm 1.56$ years. They were randomly selected from patients seeking orthodontic treatment at Outpatient clinic, Department of Orthodontics, Faculty of Dental Medicine, Al-Azhar University, Cairo (Boys).

In accordance with previous study ${ }^{(10)}$ sample size calculation was undertaken with $\mathrm{G}^{*}$ power statistical software (version 3.1, Universität Dusseldorf, Dusseldorf, Germany) based on the following preestablished parameters: an $80 \%$ power, significance 
level (alpha) $=0.05$ (two-tailed) and an effect size of 1.012. The estimated minimum sample needed to have adequate power to detect difference would be 10 patients. It was decided to increase the number of patients to 14 to compensate for any possible drop out during the course of the study.

The criteria of selection were (1) Skeletal and dental Class II malocclusion due to mandibular retrusion $\left(\mathrm{ANB} \geq 5^{\circ}, \mathrm{SNA}=82^{\circ} \pm 4, \operatorname{SNB} £ 76^{\circ}\right)$; (2)Overjet $\geq 5 \mathrm{~mm}$; (3)Normal or low FMA angle $\left(£ 28^{\circ}\right)$; (4)Complete permanent dentition, excluding the third molars; (5) Minimum or no crowding in the mandibular arch ( $\leq 3 \mathrm{~mm})$; and (6) Patients would be in post-pubertal skeletal age (stage 5 or greater) based on modified cervical vertebral maturation stages (CVMS) of Baccetti et al ${ }^{(25)}$ at the pretreatment time point; (7) No previous orthodontic treatment.

Afterward; study objectives, purpose of intervention, and the associated risks and benefits were fully explained to patients and/ or legal guardians and signed informed consents were obtained before treatment initiation.

\section{Interventions}

\section{Orthodontic records:}

The diagnosis for each patient enrolled in the study was based on detailed clinical examination and analysis of routine orthodontic records.

\section{Fixed functional appliance:}

All patients in the current study were treated by Herbst type IV appliance (Dentaurum GmbH \&Co. $\mathrm{KG}$, Germany) that consisted of a bilateral telescope mechanism attached to upper and lower jaws keeping the mandible in an anterior jumped position. ${ }^{(26)}$ The appliance was anchored by two miniplates plates (Universal reconstructive plates $2.3 \mathrm{~mm}, 4$ holes, Stryker, USA) inserted bilaterally in the mandible between the canine and first premolar teeth, ${ }^{(16,26)}$ while attached to the maxilla through dental splints utilizing orthodontic bands (Fig.1).

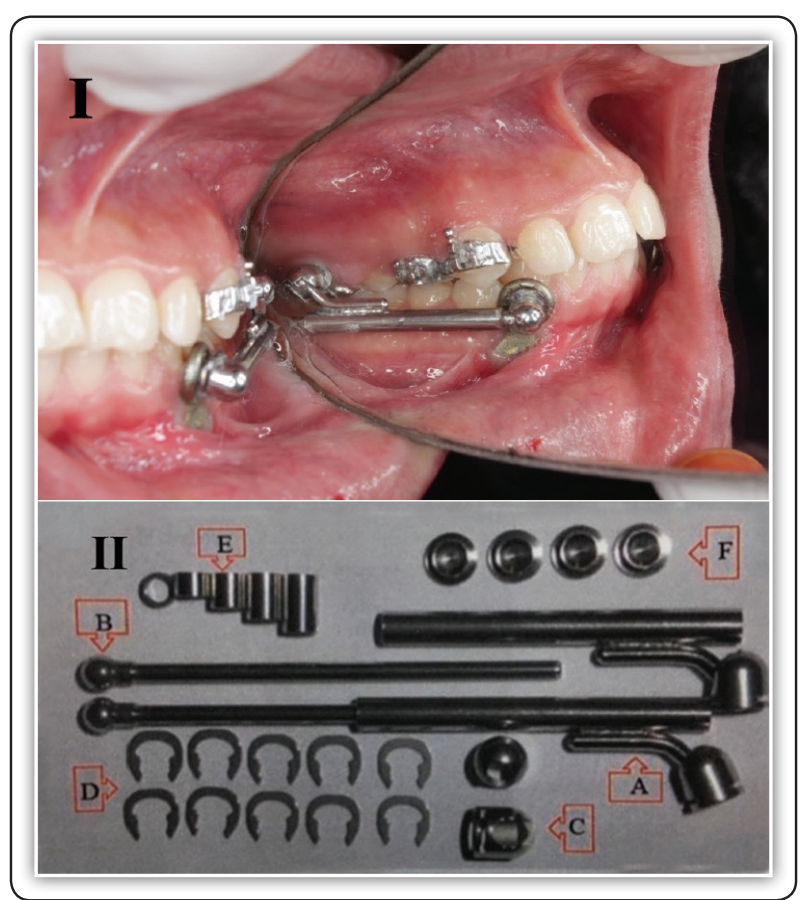

FIG (1) Herbst appliance in its place inraorally. (II)Herbst type IV components; (A) Telescopic tube with ball and socket joint, (B) Rod with ball head, (C)Coupling for ball head, (D) C-clips, (E) Spacers 5mm length, (F) Curved bases.

The reconstruction plates were pre-bended using a 3-jaw contouring and bending plier to be adapted to 3D printed (Anycubic Chiron 3D Printer, China) mandibular model that was extracted from pretreatment CBCT of each patient on the predetermined location between mandibular canine and $1^{\text {st }}$ premolar bilaterally. This step allowed proper placement and adaption on bone surface during surgery and reduced overall time of surgical procedure (Fig.2). Surgical placement of reconstruction plates was performed under local anesthesia, after performing an envelope flap extended apically to the chin level to allow unstrained placement of the reconstruction plate. (Fig.3) They were fixed by three surgical screws (diameter $2 \mathrm{~mm}$, lengths 8 and $10 \mathrm{~mm}$, Stryker, USA). 


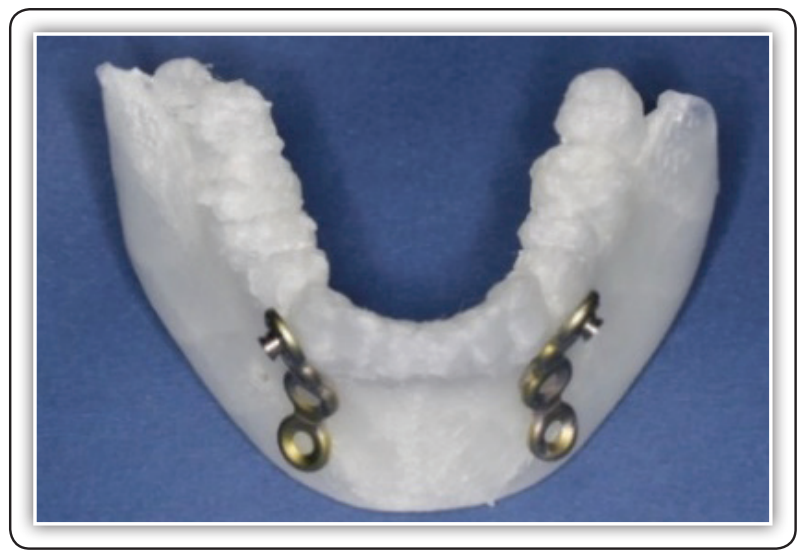

FIG (2) Pre-bended reconstruction plates adaptation over a 3D printed mandiblar model.

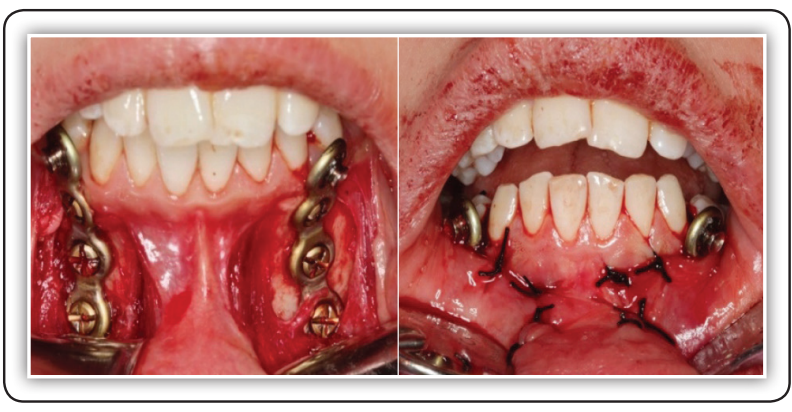

FIG (3) Reconstruction plates surgicallly placed intraorally.

\section{Maxillary dental splint fabrication:}

Maxillary dental splint fabricated by selection of proper size ready-made bands for the maxillary first premolars, first and second molars, while canine bands were custom made for each patient, then constructing palatal connecting wire and laser-welding the bands to the palatal wires was performed in addition to two transpalatal archesbetween the upper first and second molars bilaterally. The mandible was advanced to an incisal edge-to-edge position in one step advancement, however, in two patients, because of the excessive overjet, the edge to edge position seemed to be traumatic, and therefore two steps advancement about $5 \mathrm{~mm}$ for each was undertaken by adding suitable spacers ${ }^{(22)}$.

Patients were recalled one day following appliance insertion then every two weeks, to check for the followings: mucosal irritation, oral hygiene monitoring, plates' looseness, easy sliding of the telescopic rods inside the tubes, correct position of the c-clasp, and any signs of TMJ problems. The Herbst appliances were removed after nine months with the reconstruction plates through a second sur$\operatorname{gery}^{(16)}$.

After completing the first phase of FFA therapy, a complete set of records were taken and a second phase of fixed orthodontic treatment was considered for each patient according to the proposed treatment plan.

\section{Study measurements and data collection}

In addition to regular orthodontic records, Cone Beam Computed Tomographic (CBCT) scans were obtained before treatment (T1) and immediately after removal of Herbst functional appliance (T2). All CBCT images were taken with the same machine with the following exposure parameters: $20.1 \times 17.3 \mathrm{x}$ 10.2 -cm field of view, $90 \mathrm{kVp}, 12.5 \mathrm{MA}, 15$-second scan time, and $0.2 \mathrm{~mm}$ voxel size. (Planmeca ProMax® 3D Mid Imaging Unit, USA)

It is important to note that during CBCT imaging, each patient was asked to hold her breath at the end of expiration, without swallowing while the scans were taken, because the pharyngeal airway competence when awake is smallest at this time. This position was proposed to be stable with high reproducibility ${ }^{(10,21)}$.After acquisition, all data were exported and transferred in Digital Imaging and Communication in Medicine (DICOM) format via a $\mathrm{CD}$ to a personal computer for airway volume and sagittal skeletalevaluation.

\section{Pharyngeal airway assessment:}

Thiswas done through 3D volumetric construction analysis of CBCT images at $\mathrm{T} 1$ and $\mathrm{T} 2$ using software (Dolphin imaging software ver.11.95, Patterson Dental, USA).An identification number was assigned to each CBCT image prior to measurement in order to mask the patient's name and the time point during $\mathrm{CBCT}$ analysis. The CBCT images were re-matched to the patient's name after complete analysis.Serial steps were followedto 
standardize the measurements in all scans by the same assessor who was unaware about the nature of the study as follows:

The horizontal boundaries for airway analysis were P1, P2 and P3 lines that had been set parallel to Frankfort horizontal plane (FHP). The line P1 is passing through posterior nasal spine (PNS)(inferior limit of nasopharynx), the line $\mathrm{P} 2$ is passing through the uvula tip (inferior limit of velopharynx), the line P3 passing through the through the epiglottis tip (inferior limit of glossopharynx). The vertical anterior boundary of pharyngeal airway was represented by the line P4 which is perpendicular to FHP and tangent to PNS (Fig. 4) ${ }^{(24,27)}$.

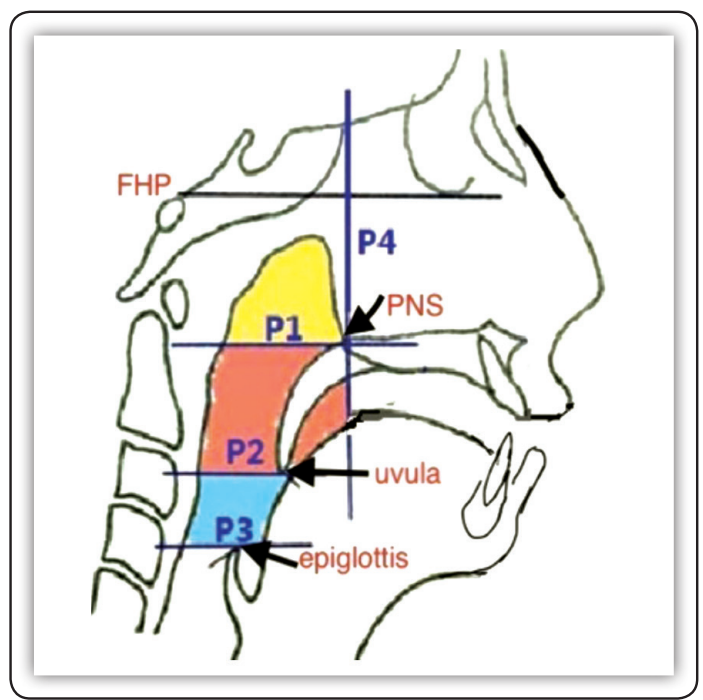

FIG (4) Diagram showing reference planes for segmentation of the pharyngeal airway used in the study.

The airway volumetric analysis was performed using the "the airway analysis" module of the software. To ensure accurate and repeatable assessment of all patients, all CBCT scans had to be oriented by the software before performing the measurements with FHP and mid-sagittal plane to ensure repeatable orientation.Although automatically performed, there are 2 interactive steps for airway segmentation; placement of initial seeds regions which grow and expands regionally limited by horizontal, vertical, and pharyngeal wall anatomical boundaries, followed by selection of sensitivity threshold which had been set at 70 unit value that was found optimum for airway segmentation that captured from a $\mathrm{CBCT}^{(28)}$.

Once segmented, the software automatically converted the segmented airway into $3 \mathrm{D}$ volumes that measured in cubic millimeters. The segmentation and 3D conversion had been made for the nasopharynx, velopharynx and glossopharynx separately as well as the total pharyngeal airway (Fig. 5).

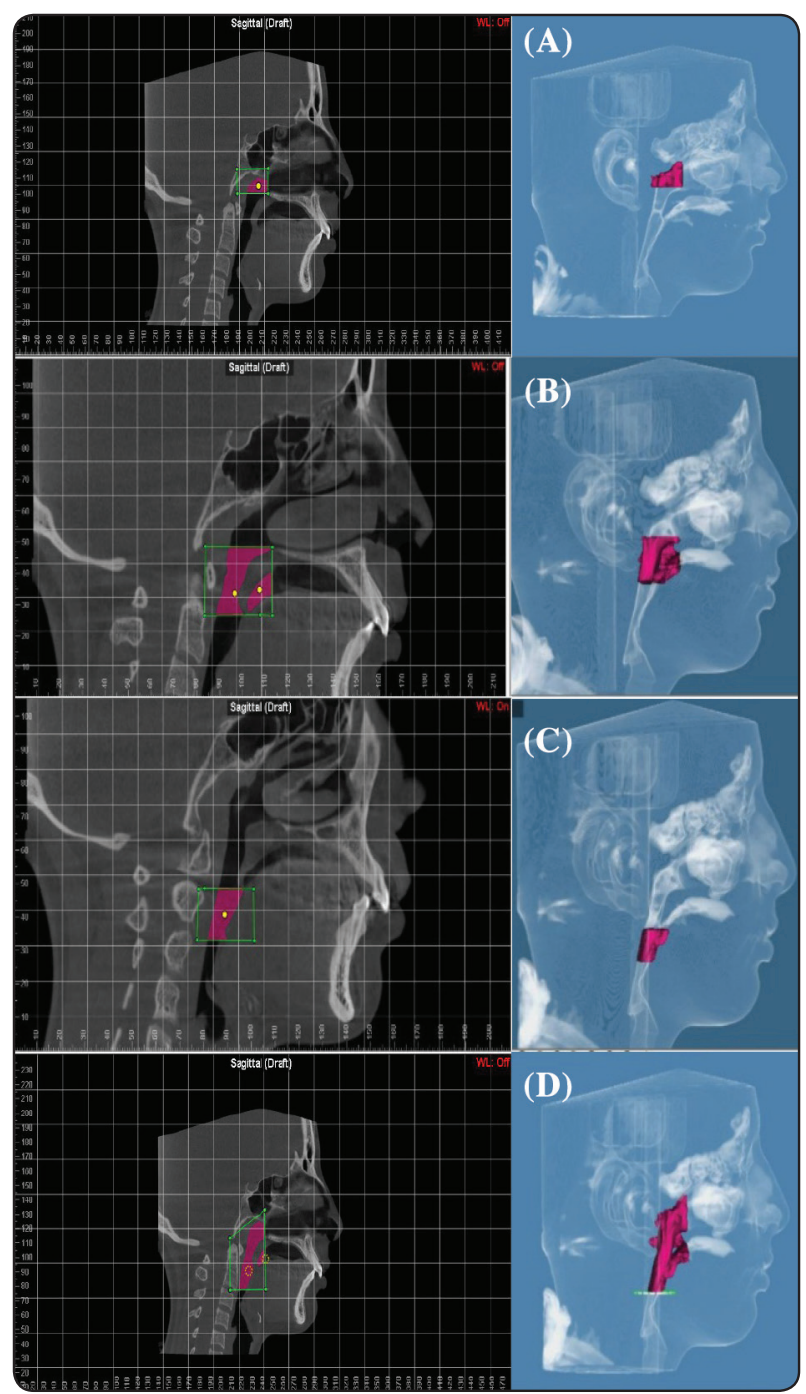

FIG (5) Assessment of pharyngeal airway volumes by segmentation and 3D volumetric analysis of CBCT images using Dolphin's airway module(colored area denotes defined airway portion of interest).(A) Nasopharynx, (B) Velopharynx, (C) Glossopharynx, (D) Total pharyngeal airway 


\section{Sagittal skeletal assessment:}

Lateral cephalometric images were reconstructed from the CBCT records to assess the possible sagittal skeletal changes based on SNA, SNB and ANB angles using both conventional cephalometric landmarks and reference planes ${ }^{(10)}$.

\section{Statistical analysis}

All data were collected and statistically analyzed by Statistical Package for Social Science software for Windows (SPSS, version 20, Inc., IBM Company, Chicago, III, USA). Descriptive statistics were displayed as means, standard deviations, and standard errors. The data were checked for normality by using Shapiro-Wilk and d'Agostino-Pearson tests. All data were normally distributed; accordingly, parametric tests were used for statistical analyses. The statistical significance was set at $\mathrm{p} \leq 0.05$.

\section{RESULTS}

\section{Drop out and loses:}

Four patients were excluded for the following reasons:two patients suffered from infection at the surgical site few days after plates' insertion that rendered the plates looseand unfunctional and had to be removed.One patient had suffered from severe TMJ pain five days after the Herbst assembly had been activated, although the amount mandibular jump had been reduced in an attempt to relieve the TMJ pain, however, it persisted and the whole assembly had to be removed. The last patient was dropped out due to failure in the solder joint connecting the Herbst buttons to the reconstruction plate and removal of plate was necessary to repair this failure. So, the final sample that analyzed was 10 patients.

\section{Analysis of error and reliability:}

Regarding the reliability of CBCT measurements in the present study, all measurements were repeated for randomly selected 4 patients (40\% of the sample) by the same examiner after 4 weeks and compared to the first measurements taken from the total sample using Interclass Correlation Coefficient. There was very high correlation between 1st and 2 nd measurements that indicated a low error of CBCT measurements.

Table 1 and figure 6 show that the nasopharyngeal airway volume reveled a non-statistically significant increase afterHerbst treatment $(\mathrm{p}=0.27)$, whereas velopharyngeal, glossopharyngeal and total airway volumes showed statistically significant increases subsequent to Herbst treatment ( $\mathrm{p}=$ $0.0004,0.002,0.0002$, respectively).

Regarding the sagittal skeletal parameters, the SNA angledid not show any significant change following Herbst treatment $(\mathrm{p}=0.742)$, even thoughthe ANB angle decreased significantly with treatment $(\mathrm{p}=0.022)$ due to the observed significant increase in SNB angle $(\mathrm{p}=0.035)$.

TABLE( 1) Descriptive statistics and comparison of pharyngeal airway volumes and sagittal skeletal parameters before (T1) and after Herbst treatment (T2) using paired sample t-test.

\begin{tabular}{|c|c|c|c|c|c|c|c|c|c|c|c|}
\hline \multirow{2}{*}{ Parameter } & \multicolumn{3}{|c|}{$\mathrm{T} 1(\mathrm{~N}=10)$} & \multicolumn{3}{|c|}{$\mathrm{T} 2(\mathrm{~N}=10)$} & \multicolumn{3}{|c|}{ Difference } & \multirow{2}{*}{ p-value } & \multirow{2}{*}{ Sig } \\
\hline & Mean & SD & $\mathrm{SE}$ & Mean & SD & SE & Mean & SD & SE & & \\
\hline Nasophayrnx $\left(\mathrm{mm}^{3}\right)$ & 5861 & 1972 & 569 & 6446 & 1725 & 498 & 585 & 762 & 508 & 0.27 & NS \\
\hline Velopharynx $\left(\mathrm{mm}^{3}\right)$ & 10549 & 3190 & 920 & 12997 & 3039 & 877 & 2448 & 1727 & 498 & 0.0004 & HS \\
\hline $\operatorname{Glosspharynx}\left(\mathrm{mm}^{3}\right)$ & 3621 & 1716 & 495 & 4729 & 1700 & 490 & 1107 & 985 & 284 & 0.002 & HS \\
\hline Total volume $\left(\mathrm{mm}^{3}\right)$ & 19701 & 4890 & 1411 & 22308 & 5322 & 1536 & 2607 & 1734 & 500 & 0.0002 & HS \\
\hline SNA $\left(^{\circ}\right)$ & 82.3 & 2.76 & 0.97 & 82.5 & 3.4 & 1.22 & 0.16 & 2.52 & 0.98 & 0.742 & NS \\
\hline $\operatorname{SNB}\left({ }^{\circ}\right)$ & 72.9 & 3.23 & 1.14 & 76.9 & 2.76 & 0.97 & 3.95 & 2.16 & 0.76 & 0.035 & $\mathrm{~S}$ \\
\hline $\operatorname{ANB}\left({ }^{\circ}\right)$ & 9.4 & 1.66 & 0.58 & 5.61 & 1.26 & 0.44 & 3.79 & 1.63 & 0.57 & 0.022 & S \\
\hline
\end{tabular}

$N=$ Number, $S D=$ Standard deviation, $S E=$ Standard error, $p=$ probability level, $T 1=$ Before treatment, T2= After Herbst treatment, $S D=$ Standard deviation, Sig = significance, NS= non-significant $p>0.05$, S significant $p \leq 0.05$, $H S=$ highly significant $p \leq 0.01$. 


\section{DISCUSSION}

Evaluation of pharyngeal airways' dimensions is of great concern to the orthodontist due to the increased prevalence of OSA in patients with constricted upper airway ${ }^{(6,7-9)}$. Interestingly, Class II cases with mandibular retrognathism had been always associated with reduced airway dimensions $^{(3-5)}$. Consequently, this study aimed to evaluate the possible volumetric pharyngeal airway changes following treatment with skeletally anchored Herbst appliance in post-pubertal Class II patients with mandibular retrognathism.

The currentresults showed that the nasopharyngeal airway volume increased by $585 \mathrm{~mm}^{3}$ but thischange was non-statistically significant $(\mathrm{P}=0.27)$. This is in agreement with Amuk et al ${ }^{(22)}$ whoreported non-significant increase in the $2 \mathrm{D}$ cephalometric evaluationof nasopharynxafter Herbst therapy in late adolescent and young adult patients. In contrast, a significant increase in the nasopharyngeal airway volume following Herbst treatment was found in MRI study of Schutz et $\mathrm{al}^{(9)}$. Nevertheless, their opposing findings may be due to preforming maxillary expansion prior to Herbst appliance insertion.

In the present research, a matched control group of untreated individuals could not be used as suggested by others. ${ }^{(26)}$ Performing such randomized controlled trials raises ethical concerns related to leaving a group of skeletal Class II subjects untreated for a lengthyperiod as well aswith needless exposure to radiation. In addition, long-term CBCT growth data among Egyptians is not available. For this reason, the treatment effects werecompared with the baseline values.

Currently, Herbst appliance treatment produced increases in velopharyngeal and glossopharyngeal airway volumes by $2448 \mathrm{~mm}^{3}$ and $1108 \mathrm{~mm}^{3}$, respectively.These findings corroborate, in part, with those of Iwsaki et al ${ }^{(10)}$ whoused Herbst appliance found $2550 \mathrm{~mm}^{3}$ and $1550 \mathrm{~mm}^{3}$ increase in velopharyngeal and glossopharyngeal airway volumes, respective- ly. The divergence concerning the glossopharyngeal region could be due to the different methodological method of their volumetric measurement in locating the inferior limit of the pharyngeal airway. In addition, Isidor et $\mathrm{al}^{(24)}$, in a retrospective CBCT study, showed significant increase in velopharyngeal and glossopharyngeal airway volume by $855 \mathrm{~mm}^{3}$ and $1167 \mathrm{~mm}^{3}$, respectively. Yet, their lower values could be due to their usageof 2 removable functional appliances for analysis. Similarly, Haskell et al achieved an average increase of $2800 \mathrm{~mm}^{3}$ in oropharyngeal volume after removable mandibular advancement device. ${ }^{(29)}$

Most of the airway studies relating airway anatomy and the craniofacial growth and development are limited because of using 2D cephalograms which cannot identify the soft tissue contour in the third dimension thus limiting evaluation of airway's areas and volumes. In this study, CBCTwas utilized in an attempt to overcome these limitations as it can generate 3D dentofacial reconstruction images that overcome the superimposition of structures with high accuracy for detailed assessment of volumetric changes ${ }^{(23,24)}$.

On the other hand, upper airway dimension has been reported to be very dependent on head posture, tongue position, and respiration phase during CBCT acquisition. Presently, the patients were asked not to swallow and to limit breathing during acquisition. Moreover, CBCT scans were taken in the upright position that is closer to the natural head position and recommended for baseline assessment of upper airway morphology and dimensions ${ }^{(23)}$.

Regarding the total pharyngeal airway volume, the current approach resulted in $2607 \mathrm{~mm}^{3}$ significant increase in the total pharyngeal airway volume. These resultsconcur with Isidor et al ${ }^{(24)}$ who used monoblock and Twin-Block removable functional appliancesandreported a significant increase of $2500 \mathrm{~mm}^{3}$ in the total pharyngeal airway. Likewise, these finding are in harmony with those of other studies ${ }^{(21,27)}$ who utilized somewhat different FFAs 
and accomplished enhancement in the pharyngeal airway volume via CBCT. Rizk et $\mathrm{al}^{(21)}$ utilized MARA appliance and reportedan increase of 2200 $\mathrm{mm}^{3}$ while Erbas et al ${ }^{(27)}$ showed increase 1700 $\mathrm{mm}^{3}$ following X-bow Forsus appliance

Although there is continuous debate regarding the effectiveness of FFAs for stimulating the mandibular growth, several reports have demonstrated successful corrections of skeletal Class II through the Herbst appliance in growing patients ${ }^{(2,8)}$.Even if there was an incremental skeletal enhancement with the rigid types of FFAs (e.g. Herbst appliance), the effect was still primarily dentoalveolar ${ }^{(30)}$.

Skeletal anchorage in conjunction with FFAs has been described to minimize these side effects, mostly with Forsus fatigue resistant device, as incorporating miniscrews ${ }^{(12,13,26,31)}$ and miniplates ${ }^{(14,15)}$. However, these reports were conflicting in regard to the achievement of actual skeletal correction of Class II malocclusion, with some of them acknowledged significant enhancement of mandibular growth ${ }^{(12,31)}$ and others disproved this effect ${ }^{(13,30)}$.

In this study, reconstructive miniplates were used for directly anchoring the Herbst appliance without bonding the mandibular arch, in line with previous reports, that supposed to offer more reliable anchorage over mini-screws upon application of orthopedic forces ${ }^{(14,16)}$. Although Herbst appliance was categorized as the most commonly used FFA, ${ }^{(2,32)}$ to the present knowledge, miniplates-anchored Herbst had not been evaluated that warrants further investigation.

In the present study, compared with base line values, an improvement in the mandibular retrognathism was evident after 9 months of miniplatesanchored Herbst therapy as demonstrated by significant increase in SNB angle and significant reductionin ANB angle. These findings of mandibular advancement are desirable outcomes of fixed functional therapy over a shorter period of treatment in non growing Class II patients and in line with other investigations in growing groups ${ }^{(8,10,12,26,32)}$.
However, the current approach suffered from some limitations. As mentioned earlier, the problematical incorporation of untreated control group with CBCT data to exclude any possible effects of remaining natural growth. As well, two surgeries were needed for miniplate insertion and removal. The additional cost is also an important shortcoming, rendering a cost-benefit analysis mandatory. Hence, engagement of this modality as an integral part of treatment for Class II patients still needs additional exploration in future studies. Moreover, supplementary 3D studies are needed to confirm the long-term influence of Herbst treatment on pharyngeal airway parameters and the stability of its outcomes.

Although a small sample size could be drawback of this study, a power analysis was used to determine the minimum desirable size. Moreover, statistical significance was achieved indicating that the treatment effect was large enough to be detected in the sample. Finally, gender limitation to females was adopted because of the recognized biological variations between males and females, rendering the validity of grouping of their outcomes could be questionable ${ }^{(16)}$. Furthermore, past researches regarding the influence of gender on airway dimensions has produced conflicting results. Abramson et al ${ }^{(33)}$ reported no effect of gender on measured airway parameters, whereas Tan et $\mathrm{al}^{(34)}$ found larger airway volumes, particularly the oropharyngeal, in males. Consequently, there is a necessitate for future studies to account for gender as a potential confounding factor.

\section{CONCLUSION}

Based on the results of the present study, pharyngeal airway volume displayed enormous enhancement following mandibular advancement via miniplate-anchored Herbst appliance which would be helpful treatment option for post-pubertal patients who have skeletal Class II malocclusion with mandibular retusion and suffering breathing disorders. 


\section{REFERENCES}

1. McNamara JA. Components of class II malocclusion in children 8-10 years of age. Angle Orthod 1981; 51:177-202.

2. Zymperdikas VF, Koretsi V, Papageorgiou SN, Papadopoulos MA. Treatment effects of fixed functional appliances in patients with Class II malocclusion: a systematic review and meta-analysis. Eur J Orthod 2016;38:113-126.

3. Ei H, Palomo J. Airway volume for different dentofacial skeletal patterns. Am J Orthod Dentofacial Orthop 2011;139:511-521.

4. Abdelkarim A. A cone beam CT evaluation of oropharyngeal airway space and its relation to mandibular position and dentocranial morphology. J World Fed Orthod 2012;1:55-59.

5. Kirjavainen M, Kirjavainen T. Upper airway dimensions in Class II Malocclusion. Angle Orthod 2017;77:10461053.

6. Katyal V, Pamula Y, Martin AJ. Craniofacial and upper airway morphology in pediatric sleep-disordered breathing: systematic review and meta-analysis. Am J Orthod Dentofacial Orthop 2013;143:20-30.

7. Sutherland K, Vanderveken OM, Tsuda H, Marklund M, Gagnadoux F. Oral appliance treatment for obstructive sleep apnea: an update. J Clin Sleep Med 2014;10: 215227.

8. Atresh A, Cebvidances L, Yatabe M, Muniz L, Nguyen T. Three-dimensional treatment outcomes in Class II patients with different vertical facial patterns treated with the Herbst appliance. Am J Orthod Dentofacial Orthop 2018;154:238-248.

9. Schutz T, Dominguez G, Hallinan M, Cunha T, Tufik S. Class II correction improves nocturnal breathing in adolescents. Angle Orthod 2011;81:222-228.

10. Iwasaki T, Takemoto Y, Inada E, Sato H, Saitoh I, Kakuno E, Kanomi R, Yamasaki Y. Three-dimensional cone-beam computed tomography analysis of enlargement of the pharyngeal airway by the Herbst appliance. Am J Orthod Dentofacial Orthop 2014;146:776-785.

11. Bermen J, Ludwig B, Ruf S. Anchorage loss due to Herbst mechanics - preventable through miniscrews? Eur J Orthod 2015;37:462-486.

12. Manni A, Pasini M, Mazzotta L, Mutinelli S, Nuzzo C, Grassi FR. Comparison between an acrylic splint Herbst and an acrylic splint miniscrew-Herbst for mandibular incisors proclination control. Int J Dent 2014;18: 87-94.
13. Aslan B, Kucukkaraca E, Turkoz C, Dincer M. Treatment effects of the Forsus Fatigue Resistant Device used with miniscrew anchorage. Angle Orthod 2014;84:76-87.

14. Unal T, Celikoglu M, Candirli C. Evaluation of the effects of skeletal anchoraged Forsus FRD using miniplates inserted on mandibular symphysis: A new approach for the treatment of Class II malocclusion. Angle Orthod 2015;85:413-419.

15. Turkkahraman H, Eliacik SK, Findik Y. Effects of miniplate anchored and conventional Forsus Fatigue Resistant Devices in the treatment of Class II malocclusion. Angle Orthod 2016;86:1026-1032.

16. Elkordy S, Abouelezz A, Fayed M, Aboulfotouh M, Mostafa Y. Evaluation of the miniplate-anchored Forsus Fatigue Resistant Device in skeletal Class II growing subjects: A randomized controlled trial. Angle Orthod 2019;89:391-403.

17. Ozbek MM, Memikoglu TU, Gogen H, Lowe AA, Baspinar E. Oropharyngeal airway dimensions and functionalorthopedic treatment in skeletal Class II cases. Angle Orthod.1998;68:327-336.

18. Yassaei S, Bahrololoomi Z, Sorush M. Changes of tongue position and oropharynx following treatment with functional appliance. J Clin Pediatr Dent 2007;31:287-290.

19. El-Bedwehi AA, Hussein FA. Al-Khalifa HN. Changes in pharyngeal airway dimensions following early treatment of Class II malocclusion by bionator appliance. AJDS 2009;12:2-13.

20. Drosen C, Bock N, Bermen J, Pancherz H, Ruf S. Longterm effects of Class II Herbst treatment on the pharyngeal airway width. Eur J Orthod 2017;39:1-8.

21. Rizk S, Kulbersh V, Al-Qawasmi R. Changes in the oropharyngeal airway of Class II patients treated with the mandibular anterior repositioning appliance. Angle Orthod 2016;86:955-961.

22. Amuk N, Kurt G, Baysal A, Turker G. Changes in pharyngeal airway dimensions following incremental and maximum bite advancement during Herbst-rapid palatal expander appliance therapy in late adolescent and young adult patients: a randomized non-controlled prospective clinical study. Eur J Orthod 2019;41:1-9.

23. Guijarro-Martinez R, Swennen GR. Cone-beam computerized tomography imaging and analysis of the upper airway: a systematic review of the literature. Int J Oral Maxillofac Surg 2011;40:1227-1237. 
24. Isidor S, Carlo G, Cornelis M, Isidor F, Cattaneo P. Threedimensional evaluation of changes in upper airway volume in growing skeletal Class II patients following mandibular advancement treatment with functional orthopedic appliances. Angle Orthod 2018;88:552-559.

25. Baccetti T, Franchi L, McNamara JA Jr. An improved version of the cervical vertebral maturation (CVM) method for the assessment of mandibular growth. Angle Orthod 2002;72:316-323.

26. Celikoglu M, Buyuk S, Ekizer A, Unal T. Treatment effects of skeletally anchored Forsus FRD EZ and Herbst appliances: A retrospective clinical study. Angle Orthod 2016;86:306-314.

27. Erbas B, Kocadereli I. Upper airway changes after Xbow appliance therapy evaluated with cone beam computed tomography. Angle Orthod 2014;84:693-700.

28. Alves M, Baratieri C, Mattos C, Brunetto D, Fontes R, Santos J. Is the airway volume being correctly analyzed? Am J Orthod Dentofacial Orthop 2012;141:657-661 .

29. Haskell JA, McCrillis J, Haskell BS, Scheetz JP, Scarfe WC, Farman AG. Effects of mandibular advancement device (MAD) on airway dimensions assessed with cone-beam computed tomography. Semin Orthod 2009;15:132-158.
30. Elkordy S, Aboelnaga A, Fayed M, Aboulfotouh M, Abou El-Ezz A. Can the use of skeletal anchors in conjunction with fixed functional appliances promote skeletal changes? A systematic review and meta-analysis. Eur J Orthod 2016;38:532-545.

31. Celikoglu, M, Unal T, Bayram M, Candirli C. Treatment of a skeletal Class II malocclusion using fixed functional appliance with miniplate anchorage. Eur J Dent 2014; 8:276-280

32. Yang X, Zhu Y, Long H, Zhou Y, Jian F, Ye N, et al. The effectiveness of the Herbst appliance for patients with Class II malocclusion: a meta-analysis. Eur J Orthod 2016;38:324-333.

33. Abramson Z, Susarla S, Troulis M, Kaban L. Age-related changes of the upper airway assessed by 3-dimensional computed tomography. J Craniofac Surg 2009;20: 657-663.

34. Tan R, Pangrazio V, Kulbersh R, Al-Qawasmi R. CBCT evaluation of changes in the total pharyngeal airway in subjects from seven to eighteen years. In: Park, J., ed. Computed Tomography: New Research. New York: Nova Science; 2013:393-400. 